\title{
2D shear wave elastography (SWE) performance versus vibration-controlled transient elastography (VCTE/fibroscan) in the assessment of liver stiffness in chronic hepatitis
}

\author{
Ahmed M. Osman *iD, Ahmed El Shimy and Mohamed M. Abd El Aziz
}

\begin{abstract}
Background: The assessment of liver stiffness and the degree of fibrosis are important factors affecting the management strategy. Multiple non-invasive tools are now available to offer an adequate alternative to biopsy. In this study, we tried to compare the performance of 2D shear wave elastography (SWE) to the transient elastography/fibroscan as a non-invasive tool in the prediction of liver stiffness. This is a prospective study of 215 patients confirmed by serology to have positive virus C or B infection. 2D SWE was done followed by vibrationcontrolled transient elastography (VCTE) known as fibroscan at the same session. Biopsy results were collected.

Results: The mean age was 51.07 years \pm 6.07 SD. Five cases were excluded due to insufficient data. Fibroscan failed in 30 cases out of 210 cases (failure rate of 14.3\%) compared with only 12 patients (6.7\% failure rate) while using SWE. Only 180 patients completed the study to the result analysis. SWE results showed significant agreement to the fibroscan results with $86.7 \%$ agreement with a tendency for overestimation of the degree of fibrosis (11.7\%). The efficacy of SWE was the highest during the assessment of patients with F0 (98.9\%), F1 (97.8\%), and F4 (93.3\%) respectively and relatively low in F2 (92.8\%) and F3 (90.6\%).

Conclusion: 2D SWE is a relatively recent non-invasive tool in the assessment of liver fibrosis grading which can be used as an alternative to the fibroscan with almost similar diagnostic performance especially when fibroscan is not capable to obtain adequate results such as in obesity and ascites.
\end{abstract}

Keywords: Chronic liver disease, Liver stiffness, Shear wave elastography, Fibroscan, Transient elastography

\section{Key points}

- Chronic liver disease is one of the commonest chronic diseases worldwide.

- The degree of fibrosis is important to determine the treatment strategy.

- SWE and fibroscan are non-invasive tools for liver fibrosis grading.

- SWE offers almost similar diagnostic accuracy as fibroscan with overestimation tendency.

\section{Background}

Chronic liver disease (CLD) is one of the most common chronic diseases worldwide with multiple etiological factors and high morbidity and mortality rates [1]. CLD caused by multiple factors including alcohol, viral hepatitis, drug induced, auto-immune diseases, and obesity with all these factors leads to liver fibrosis/cirrhosis which may end to liver cell failure and death [2]. Viral hepatitis is one of the commonest causes of CLD especially in Egypt with hepatitis $C$ comes on top of the causes of CLD in Egypt [3].

\footnotetext{
*Correspondence: Dr_osman80@yahoo.com; Dr_aosman@med.asu.edu.eg
} Faculty of Medicine, Ain Shams University, Cairo, Egypt 
The management and treatment strategy of CLD depends on the clinical status of the patient, laboratory liver profile, and the degree of liver fibrosis [4]. Liver biopsy was considered the gold standard for assessment of the degree of liver fibrosis, but it carries a lot of risks being painful, expensive, and risk of hemorrhage comes on top of these complications [5].

As a result of liver biopsy complications, the search for non-invasive tools for assessment and grading of liver fibrosis is rising with transient elastography (TE) or fibroscan becomes the commonest non-invasive tool used as an alternative for biopsy. It is a non-invasive, bedside, and rapid test $[2,6]$.

TE probe uses a mechanical vibration that creates shear wave within the liver parenchyma and also ready to read the velocity reflected from the liver surface which shows changes according to the degree of liver fibrosis, then giving a measurement reflecting the degree of liver stiffness which is displayed in kilopascal $(\mathrm{kPa})[7,8]$. TE or fibroscan has some limitations when used in patients with ascites, morbid obesity, and massive pleural effusion [9].

2D shear wave elastography (SWE) is relatively recent tool in the era of liver fibrosis grading as a non-invasive tool which uses the usual 2D US probe, yet with production of a focused acoustic beam to generate shear wave within the liver with the degree of liver fibrosis reflected upon the speed of this wave within the liver parenchyma and affects the degree of reflected wave. The same probe can track the reflected wave then calculate the degree of liver fibrosis using the machine software which is presented with meter per second $[10,11]$.

In this study, we tried to assess the diagnostic performance of SWE as a non-invasive tool in the detection of the degree of liver stiffness and fibrosis compared to the TE/fibroscan in patients with known CLD.

\section{Methods \\ Patients}

This was a prospective study conducted on randomized selected 215 patients known to have chronic hepatitis infection either hepatitis C or hepatitis B and came for liver fibrosis grading as pre-therapeutic assessment or follow-up during the management course. Each patient did the usual B-mode ultrasound followed by 2D SWE then TE or fibroscan which was done in the same session. The study was conducted over the period from March 2019 to October 2019. Written informed consent was taken from all patients to use their results data according to the ethical committee regulations.

\section{Inclusion criteria}

All patients with chronic hepatitis and proved by laboratory data to have positive viral $\mathrm{C}$ or $\mathrm{B}$ infection. No age or sex predilection. Available hepatic biopsy results with histopathological fibrosis scoring with a maximum of three months before the scan time.

\section{Exclusion criteria}

We excluded patients with congestive heart disease, acute hepatitis, hepatic, or portal vein thrombosis or anomalies, and any patient with hepatic focal lesion or history of a hepatic interventional procedure as radiofrequency ablation (RF) or chemoembolization. Also, patients with unavailable hepatic biopsy results or biopsies more than 3 months before the scan time.

\section{Patient preparation}

The patient underwent fasting for 4-6 h. An adequate full history was taken. A documented laboratory result for hepatitis markers was available. A previous biopsy result also needed.

\section{Technique \\ US machine}

LOGIQ S8 XDclear 2.0 with vibration controlled TE probe and the 2D SWE software. General Electric (GE) company, USA.

\section{The technique for SWE}

All patients were placed supine or left semi-lateral decubitus with the 2D convex probe placed on the midclavicular line or anterior axillary line in the intercostal spaces until obtaining the most adequate window for the liver. The right lobe was the selected region of the liver with a distance of about $2 \mathrm{~cm}$ to the nearby capsule and devoid of a large blood vessel as we could. The patients were asked to hold breathing to minimize breathing motion artifacts. Then, SWE was initiated for about $5 \mathrm{~s}$ on the selected liver area through which about two to three frames of SWE were obtained. A region of interest (ROI) was applied inside each frame of SWE away from any visualized artifacts to obtain the best quantitative measurements. Twelve adequate measurements were needed. The $V$ median/IQR ratio was considered to be < $25 \%$ to ensure adequate results and readings.

Table 1 The interpretation of the SWE results by meter per second and the liver fibrosis staging using Metavir scoring

\begin{tabular}{lll}
\hline Liver fibrosis staging & Metavir score & $\mathrm{m} / \mathrm{s}$ \\
\hline Normal & F0 & $<1.47 \mathrm{~m} / \mathrm{s}$ \\
Normal-mild & F1 & $1.47-1.48 \mathrm{~m} / \mathrm{s}$ \\
Mild-moderate & F2 & $1.48-1.64 \mathrm{~m} / \mathrm{s}$ \\
Moderate-severe & F3 & $1.64-1.76 \mathrm{~m} / \mathrm{s}$ \\
Cirrhosis & F4 & $>1.76 \mathrm{~m} / \mathrm{s}$ \\
\hline
\end{tabular}




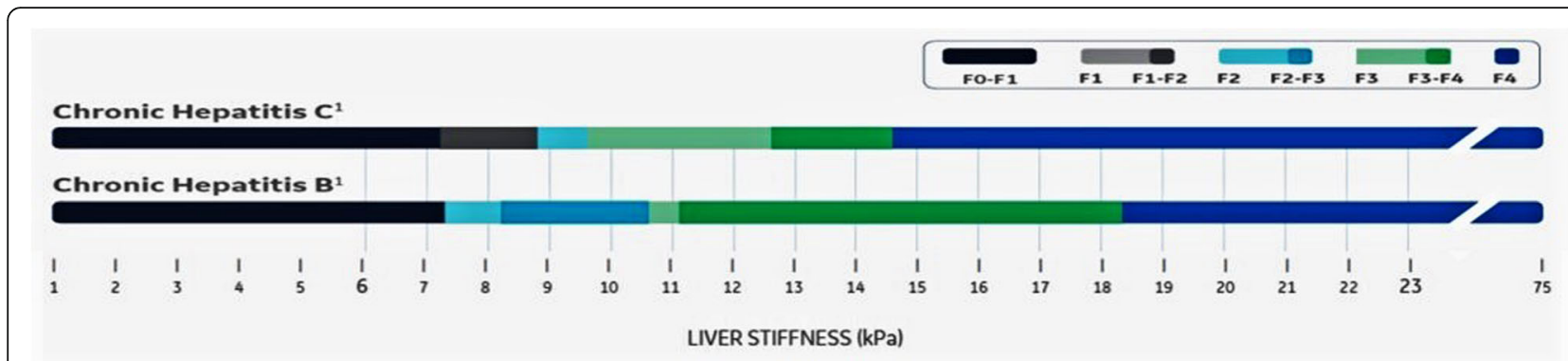

Fig. 1 The interpretation of TE or fibroscan results by kilopascal and the liver fibrosis staging (quoted from GE healthcare documents, 2017)

\section{The technique for VCTE or fibroscan}

All patients were placed on a supine position with elevated arms resting above the head level. B-mode was used to select the best intercostal space level at the midaxillary line (best at the level of the xiphoid process) with the best visualization field for the liver. We activated the fibroscan probe and applied it at the selected point perpendicular to the skin surface. An adequate amount of gel was considered, and appropriate compression pressure was applied known by the pressure indicator green light appeared on the screen. Ten measurements were taken to obtain the median number in kilopascal. IQR/median ratio was considered to be < $25 \%$ to ensure adequate results and readings.

\section{Image interpretation}

The three authors are well trained on elastography techniques with at least 3 years of experience and all were blinded by the tissue biopsy result. Each patient did the SWE first with one of the authors followed by TE by another one who was blinded about the result of SWE. All results of each patient were collected. The measurements of SWE were expressed by meter per second and interpreted into liver fibrosis staging according to Metavir scoring (Table 1). The SWE speed converted to kilopascal using Young's formula $\left(\mathrm{kPa}=3 \mathrm{pv}^{2}\right)$ with $p=$ tissue density which is always considered $1000 \mathrm{~kg} / \mathrm{m}^{3}$ and $v=$ speed of SWE [12].

Regarding the TE or fibroscan, the results were expressed by kilopascal which also interpreted into liver fibrosis staging according to Fig. 1 [13-16]. SWE results and the fibroscan results were compared to the liver tissue biopsy which was considered as the gold standard reference for liver fibrosis staging. Then, the SWE results were compared to the TE or fibroscan results while used as reference values.

\section{Analysis of data}

The analysis of data was done using IBM SPSS statistics (v. 25.0, IBM Corp., USA, 2017-2018) was used for data analysis. Data were expressed as mean \pm SD for quantitative parametric measures in addition to both number and percentage for categorized data.

The following tests were done:

1. Chi-square test to study the association between every 2 variables or comparison between 2 independent groups as regards the categorized data. The probability of error at 0.05 was considered sig., while at 0.01 and 0.001 are highly sig.

2. Diagnostic validity test includes sensitivity, specificity, and positive and negative predictive value as well as the efficacy.

\section{Results}

This is a study conducted on 215 randomized selected patients with known CLD secondary to chronic hepatitis C or B infection. Only 180 patients completed the study to the

Table 2 The results of the fibroscan fibrosis score between the selected population compared to the tissue biopsy

\begin{tabular}{|c|c|c|c|c|c|c|c|c|}
\hline & & & \multicolumn{5}{|c|}{ Fibroscan (VCTE) fibrosis score } & \multirow[t]{2}{*}{ Total } \\
\hline & & & F0 & F1 & F2 & F3 & F4 & \\
\hline \multirow[t]{5}{*}{ Liver biopsy score } & FO & Count & 33 & 2 & 0 & 0 & 0 & $35(19.4 \%)$ \\
\hline & F1 & Count & 2 & 26 & 1 & 1 & 1 & $31(17.2 \%)$ \\
\hline & $\mathrm{F} 2$ & Count & 1 & 1 & 28 & 2 & 0 & $32(17.8 \%)$ \\
\hline & F3 & Count & 0 & 1 & 0 & 32 & 3 & $36(20 \%)$ \\
\hline & F4 & Count & 0 & 0 & 1 & 1 & 44 & $46(25.6 \%)$ \\
\hline \multirow[t]{2}{*}{ Total } & & Count & 36 & 30 & 30 & 36 & 48 & 180 \\
\hline & & $\%$ & $20 \%$ & $16.7 \%$ & $16.7 \%$ & $20 \%$ & $26.7 \%$ & $100.0 \%$ \\
\hline
\end{tabular}


Table 3 The results of the SWE fibrosis score between the selected population compared to the tissue biopsy

\begin{tabular}{|c|c|c|c|c|c|c|c|c|}
\hline & & & \multicolumn{5}{|c|}{ 2D SWE fibrosis score } & \multirow[t]{2}{*}{ Total } \\
\hline & & & F0 & F1 & F2 & F3 & F4 & \\
\hline \multirow[t]{5}{*}{ Liver biopsy score } & FO & Count & 32 & 2 & 1 & 0 & 0 & $35(19.4 \%)$ \\
\hline & F1 & Count & 1 & 24 & 1 & 3 & 2 & $31(17.2 \%)$ \\
\hline & $\mathrm{F} 2$ & Count & 1 & 1 & 27 & 0 & 3 & $32(17.8 \%)$ \\
\hline & F3 & Count & 0 & 1 & 0 & 28 & 7 & $36(20 \%)$ \\
\hline & F4 & Count & 0 & 0 & 0 & 0 & 46 & $46(25.6 \%)$ \\
\hline \multirow[t]{2}{*}{ Total } & & Count & 34 & 28 & 29 & 31 & 58 & 180 \\
\hline & & $\%$ & $18.9 \%$ & $15.6 \%$ & $16.1 \%$ & $17.2 \%$ & $32.2 \%$ & $100.0 \%$ \\
\hline
\end{tabular}

analysis level. Five patients were excluded due to unavailable documented data about the hepatitis viral infection. Thirty patients representing $14.3 \%$ of cases were excluded due to failed fibroscan (19 patients due to obesity and 11 patients due to the presence of ascites). Eighteen of them showed successful SWE examination, while 12 patients also failed to do SWE (failure rate $=6.7 \%$ ).

The mean age of the selected patients was 51.07 years \pm 6.07 years SD. One hundred seventeen patients out of 180 were male representing $65 \%$ of the cases. Cases with positive virus $\mathrm{C}$ infection were 148 patients (82.2\%), while cases with positive virus B infection were only 32 patients (17.8\%). All the patients had available biopsy results with the median time between the biopsy and the scan time was 52 days.

Comparing the results of fibroscan and SWE with tissue biopsy showed an almost similar degree of agreement with no significant difference between the two techniques when compared with the biopsy results. However, SWE showed a higher incidence of overestimation (Tables 2 and 3). The agreement of fibroscan reached $90.6 \%$ compared with $87.2 \%$ of SWE. The degree of overestimation showed $5.6 \%$ in fibroscan while it was $10.6 \%$ in SWE. The degree of underestimation was $3.8 \%$ and $2.2 \%$ for fibroscan and SWE respectively. SWE showed a higher incidence of mismatch between patients with F4 (Table 3).

The diagnostic accuracy of both techniques showed no significant difference when compared to the liver biopsy results with almost similar and close efficacy results as seen in Table 4.
Regarding fibroscan (VCTE) fibrosis score, patients with F4 were the comments representing 48 patients (26.7\%) followed by F3 and F0 with both of them found in 36 patients (20\%). F2 and F1 were the least with each found between 30 patients (16.7\%) (Table 5).

When comparing the results of SWE to the fibroscan results, we found $86.7 \%$ agreement with $11.7 \%$ overestimation and $1.7 \%$ underestimation. $P$ value was $<0.001$ denoting a highly significant correlation between the results of SWE and the results of fibroscan.

The highest incidence of mismatch was found between patients with F3 fibroscan score (11 cases) followed by patients with F2 fibroscan score (7 cases), while the mismatch was the least between patients with fibroscan score F0 and F1 (2 and one respectively) (Table 5).

The SWE tends to overestimate the results fibrosis score when compared to the fibroscan with the highest degree of overestimation found at F3 and F2 patients. SWE shows high efficacy in all degrees of fibrosis with the lowest found at F3 and F2, while the highest efficacy found at F0 and F1 (Figs. 2, 3, 4 and 5) (Table 6).

The duration of the fibroscan was $5.3 \pm 2.2 \mathrm{~min}$, while it was $5.4 \pm 2.5 \mathrm{~min}$ for SWE with no significant difference between the two techniques regarding the duration of the technique.

\section{Discussion}

CLD secondary to chronic hepatitis infection is one of the most common chronic debilitating diseases in Egypt, and the assessment of the degree of liver fibrosis is important in patient management. Recently, the liver

Table 4 The diagnostic validity of fibroscan (TE) and SWE compared to tissue biopsy at different fibrosis scores

\begin{tabular}{|c|c|c|c|c|c|c|c|c|c|c|c|}
\hline & \multicolumn{3}{|l|}{ FO } & \multicolumn{2}{|l|}{ F1 } & \multicolumn{2}{|l|}{ F2 } & \multicolumn{2}{|l|}{ F3 } & \multicolumn{2}{|l|}{$\mathrm{F} 4$} \\
\hline & $\overline{T E}$ & & SWE & $\overline{\mathrm{TE}}$ & SWE & $\overline{\mathrm{TE}}$ & SWE & $\overline{T E}$ & SWE & $\overline{\mathrm{TE}}$ & SWE \\
\hline Sensitivity (\%) & & 94.3 & 91.4 & 83.9 & 77.4 & 87.5 & 84.3 & 88.9 & 77.8 & 95.7 & 100 \\
\hline Specificity (\%) & & 97.9 & 98.6 & 97.3 & 97.3 & 98.6 & 98.6 & 97.2 & 97.9 & 97 & 91 \\
\hline NPV (\%) & & 98.6 & 97.9 & 96.7 & 95.4 & 97.3 & 96.7 & 97.2 & 94.6 & 98.5 & 100 \\
\hline PPV (\%) & & 91.6 & 94.1 & 86.7 & 85.7 & 93.3 & 93.1 & 88.9 & 90.3 & 91.7 & 97.3 \\
\hline Efficacy (\%) & & 97.2 & 97.2 & 95 & 93.9 & 96.7 & 96.1 & 95.6 & 93.9 & 96.7 & 93.3 \\
\hline
\end{tabular}


Table 5 The results of fibrosis score between the selected population using both fibroscan and SWE technique with the fibroscan results used as the reference

\begin{tabular}{|c|c|c|c|c|c|c|c|c|}
\hline & & & \multicolumn{5}{|c|}{ Fibroscan (VCTE) fibrosis score } & \multirow[t]{2}{*}{ Total } \\
\hline & & & F0 & F1 & F2 & F3 & F4 & \\
\hline \multirow[t]{5}{*}{ SWE fibrosis score } & F0 & Count & 34 & 0 & 0 & 0 & 0 & $34(18.9 \%)$ \\
\hline & F1 & Count & 1 & 27 & 0 & 0 & 0 & $28(15.6 \%)$ \\
\hline & F2 & Count & 1 & 3 & 23 & 2 & 0 & $29(16.1 \%)$ \\
\hline & F3 & Count & 0 & 0 & 5 & 25 & 1 & $31(17.2 \%)$ \\
\hline & F4 & Count & 0 & 0 & 2 & 9 & 47 & $58(32.2 \%)$ \\
\hline \multirow[t]{2}{*}{ Total } & & Count & 36 & 30 & 30 & 36 & 48 & 180 \\
\hline & & $\%$ & $20 \%$ & $16.7 \%$ & $16.7 \%$ & $20 \%$ & $26.7 \%$ & $100.0 \%$ \\
\hline \multicolumn{9}{|l|}{ Chi-square tests } \\
\hline & & & Value & $P$ & & & & \\
\hline Pearson chi-square & & & 514.551 & 0.000 & & & & \\
\hline
\end{tabular}

biopsy no longer becomes the main tool for liver fibrosis staging to avoid its complications and hazard especially with the advanced progress in the non-invasive ultrasound tools. Different US elastography techniques now became essential in the management of patients with CLD and offer an adequate alternative to biopsy according to the World Federation for the US in medicine and biology guidelines [17].

TE or fibroscan is considered one of the most used alternatives for biopsy and already put in patient's management algorithms in most of the European countries. However, some limitation was observed with the use of $\mathrm{TE}$ or fibroscan regarding obesity and ascites $[7,18]$.

In our study, the failure rate of fibroscan reached about $14.3 \%$ secondary to two reasons namely the ascites and patient's obesity. Out of fibroscan failed cases, 18 cases were examined successfully using SWE with adequate results confirmed with $\mathrm{IQR} /$ median ratio $<25 \%$, while the rest 12 also failed by SWE with a failure rate of SWE $=6.7 \%$. This is already consistent with similar studies as Castera et al [19] who reported a failure rate of fibroscan reaching $20 \%$ with overall 5 years experience with patient's obesity was considered one of the most important causes of technique failure. Foucher et al [20] recorded a less failure rate of fibroscan $6.2 \%$ which is much lower than our result, but they found also that high body mass index was the commonest failure cause. Zeng et al [21] recorded less failure rate of SWE compared with fibroscan but with lesser incidence compared with our study being $7 \%$ regarding the fibroscan and $1.9 \%$ regarding the SWE. The difference in failure rate taking into consideration the obesity being the commonest cause may vary between the different studies depending on the race and their body mass configuration.

The lower failure rate of SWE can be explained by the simultaneous B-mode visualization available during the SWE technique which provides the opportunity to select the proper site for reading away from the ascites interface and can avoid areas of obesity which was more common in the axillary region rather than the anterior chest wall in our patients. This is in controversy to the fibroscan which depends on the application of the probe

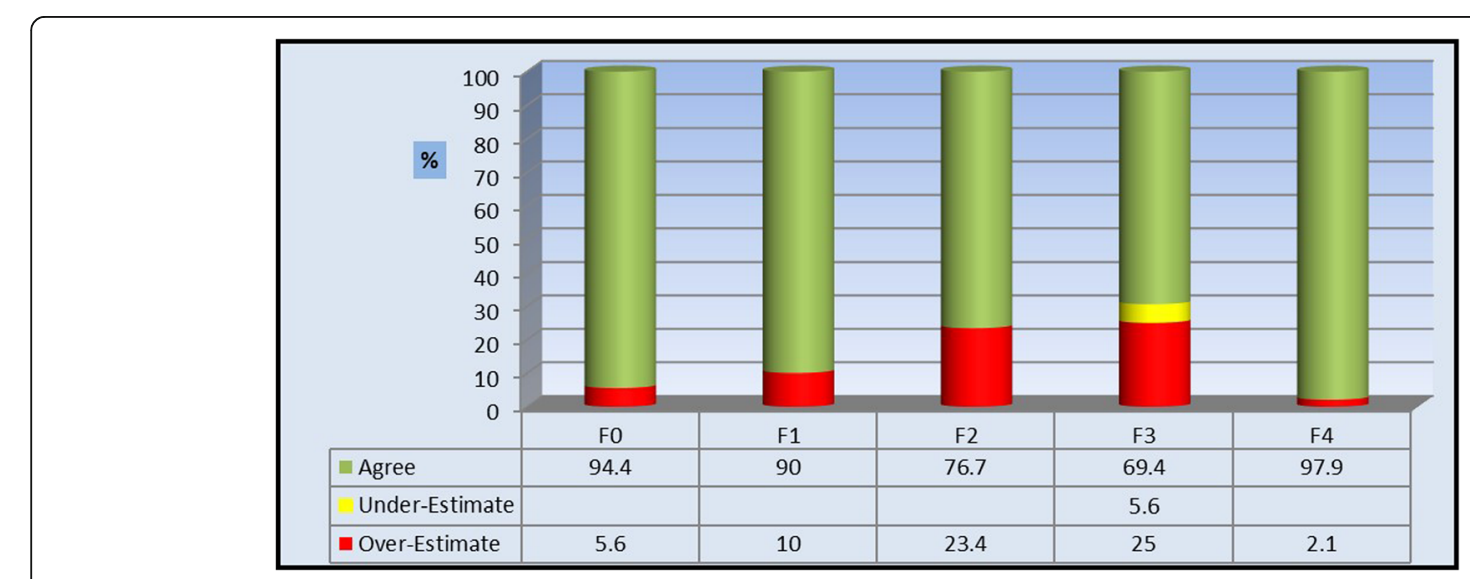

Fig. 2 The degree of agreement and the mismatch incidence between the SWE fibrosis score compared to the fibroscan fibrosis score 


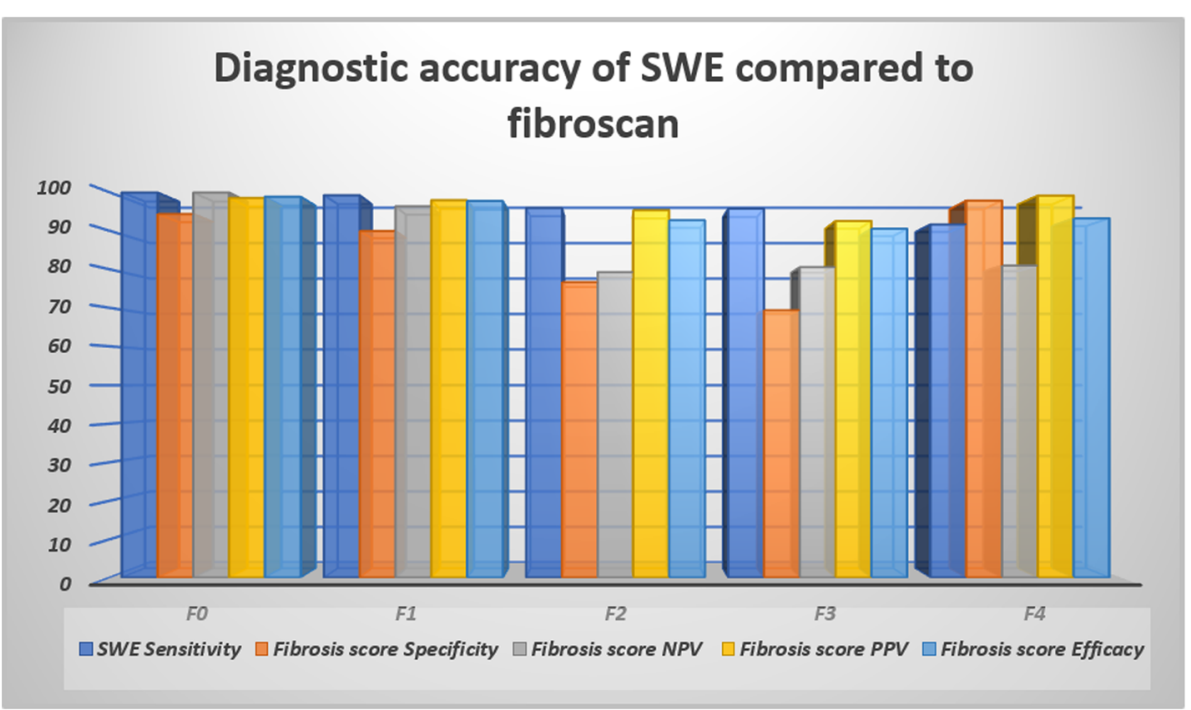

Fig. 3 The diagnostic accuracy of SWE compared to the TE fibroscan when using the TE fibroscan results as a reference

blindly on a specific anatomical level on the patient's body at the midaxillary level which showed more fat level rather than the anterior chest wall.

Our methodology was almost similar to O'Hara et al [1], Ryu et al [22], and Roccarina et al [23] who also compared the performance of SWE compared to TE with TE results was the reference values. However, their study was on a fewer sample volume compared with our study which included 180 patients. O'Hara et al [1] results were similar to our study and they concluded that SWE showed almost similar accuracy as TE, but SWE showed a tendency to overestimate the TE results.

Multiple studies $[4,10,24,25]$ tried to assess the accuracy of SWE compared with TE/fibroscan, yet they used the liver biopsy as a reference which was also applied in our study.

SWE is a recent modality used for fibrosis assessment which allows real B-mode visualization of the selected area with a larger area of selection under analysis compared with fibroscan. In this study, we assessed the diagnostic performance of 2D SWE compared with the usual $\mathrm{TE}$ or fibroscan in the assessment of liver fibrosis in patients with CLD secondary to chronic hepatitis infection. We found high significant agreement between the results of SWE and the results of fibroscan with high diagnostic performance and efficacy results between different $\mathrm{F}$ score results obtained by fibroscan with $11.7 \%$ overestimation tendency noted mainly between the patients with F3 and F2 (Tables 5 and 6 and Fig. 2).

Ali Z and colleagues [4] concluded a strong correlation and agreement between SWE and TE results with Kendalls $t-b$ was 0.902 , Spearman's $p$ value was 0.947 , and the weighted $k$ test value was 0.873 .

Leung et al [24] compared SWE results to liver biopsy with $85 \%$ and $92 \%$ SWE sensitivity and specificity respectively in the diagnosis of liver fibrosis as well as $97 \%$ and 93\% sensitivity and specificity in the diagnosis of liver cirrhosis, and this was close to our results as seen in Table 4 .

Tada et al [10] and Ferraioli et al [25] stated that SWE can be used similar to TE in the assessment of liver fibrosis. Deffieux et al [26] found that the results of SWE are almost equal or even better than the results of TE.

Zeng et al [21] compared the SWE and TE to liver biopsy in patients with chronic hepatitis B infection and

Table 6 The diagnostic validity of SWE compared to the fibroscan at different fibrosis scores

\begin{tabular}{|c|c|c|c|c|c|c|c|}
\hline & & & F0 & F1 & $\mathrm{F} 2$ & F3 & $\mathrm{F} 4$ \\
\hline \multirow[t]{5}{*}{ SWE fibrosis score } & Sensitivity & & 100 & 99.3 & 96 & 95.8 & 91.7 \\
\hline & Specificity & & 94.4 & 90 & 76.7 & 69.4 & 97.9 \\
\hline & NPV & & 100 & 96.4 & 79.3 & 80.6 & 81 \\
\hline & PPV & & 98.6 & 98 & 95.4 & 92.6 & 99.2 \\
\hline & Efficacy & & 98.9 & 97.8 & 92.8 & 90.6 & 93.3 \\
\hline \multirow[t]{2}{*}{ Pearson chi-square } & & Value & 167.671 & 151.883 & 97.674 & 86.084 & 129.346 \\
\hline & & P & 0.000 & 0.000 & 0.000 & 0.000 & 0.000 \\
\hline
\end{tabular}




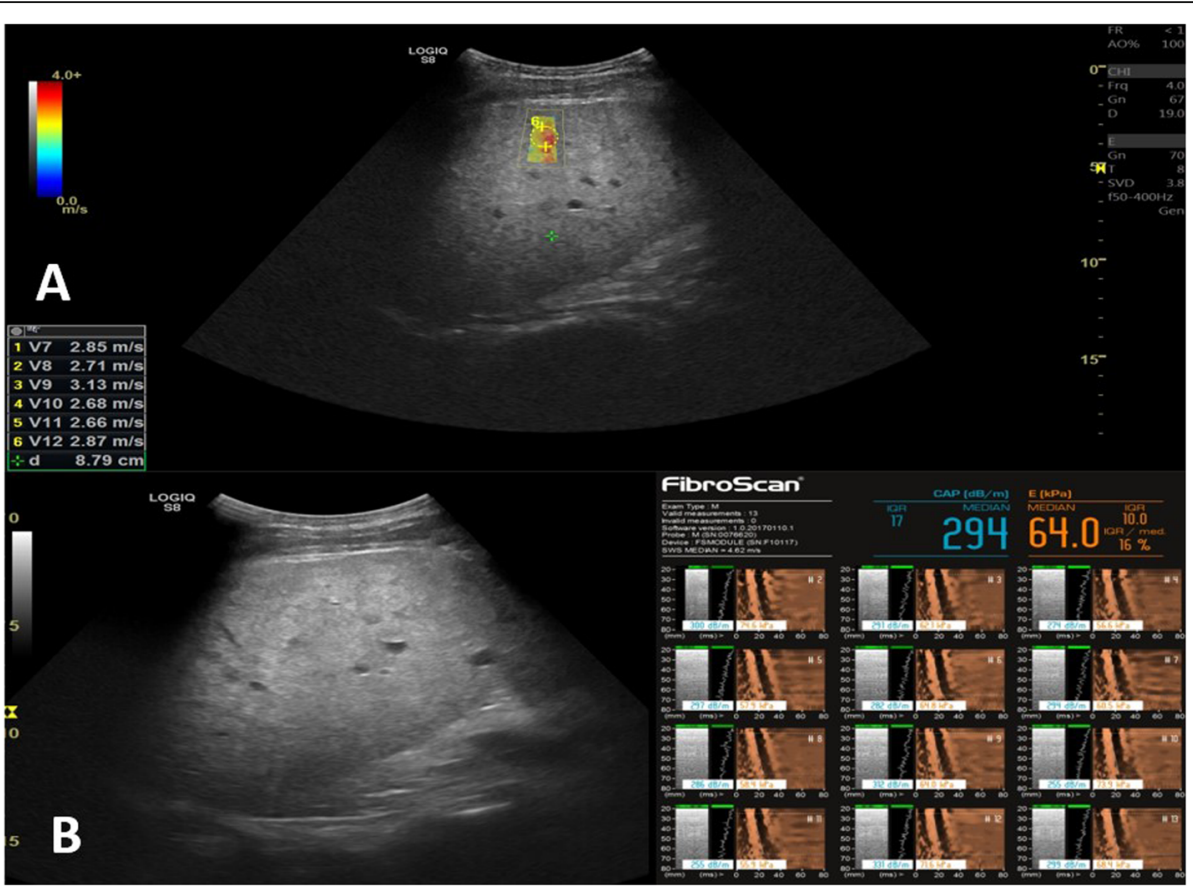

Fig. 4 A male patient 52 years old with chronic hepatitis C infection on follow-up. a SWE revealed median velocity $=2.62 \mathrm{~m} / \mathrm{s}$ and $\mathrm{V}$ median/IQR $=14.6 \%$ consistent with F4 according to Metavir score. $\mathbf{b}$ Fibroscan was done for the same patient and revealed $\mathrm{kPa}=64 \mathrm{and} I \mathrm{IQR} / \mathrm{median}=16 \%$ consistent with F4

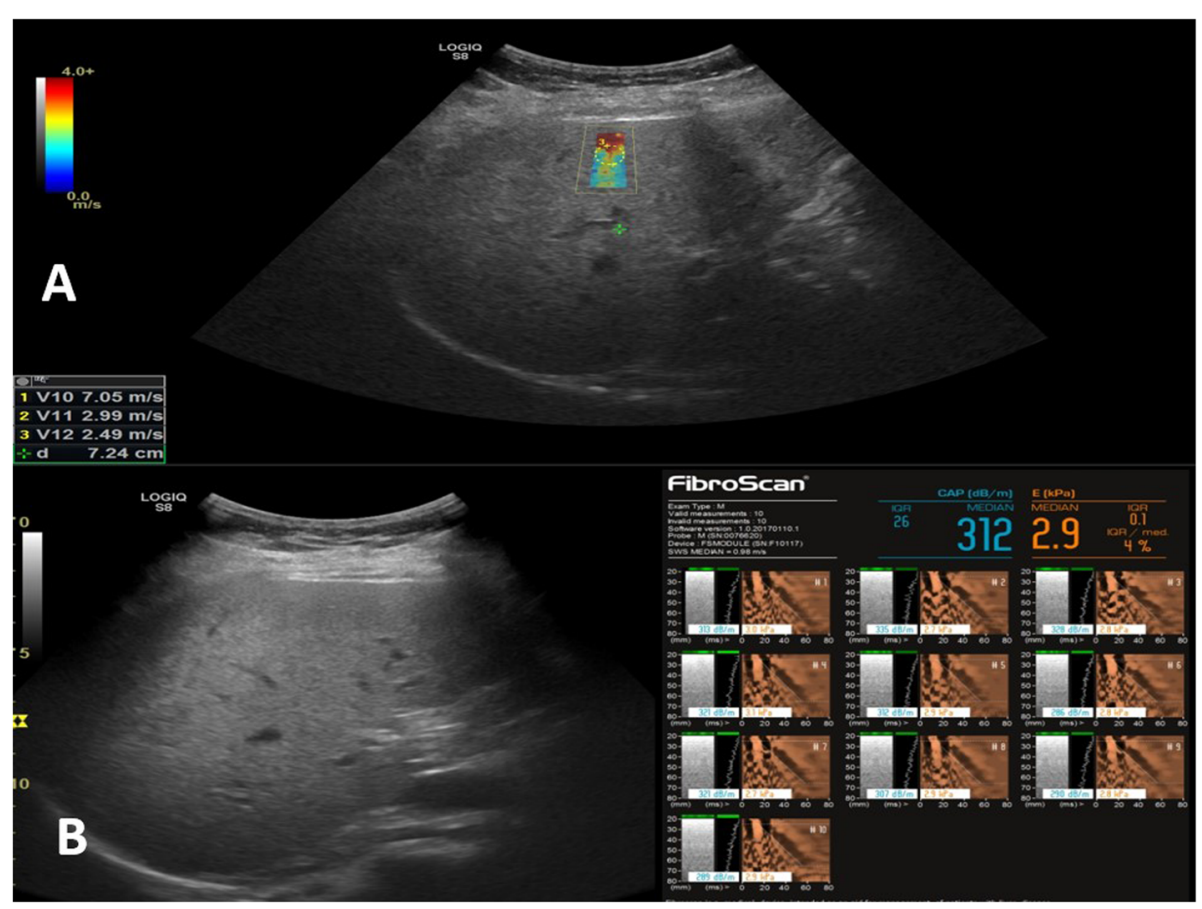

Fig. 5 A female patient 48 years old with chronic hepatitis B infection on follow-up. a SWE revealed median velocity $=1.54 \mathrm{~m} / \mathrm{s}$ and $V \mathrm{median} /$ $\mathrm{IQR}=23 \%$ consistent with F2 according to Metavir score. $\mathbf{b}$ Fibroscan was done for the same patient and revealed $\mathrm{kPa}=2.9 \mathrm{and} \mathrm{IQR} / \mathrm{median}=$ $4 \%$ consistent with FO 
found SWE had a higher rate of reliability $98.1 \%$ than TE (93\%). They found also a strong correlation between SWE and TE with no difference between the area under ROC curves of SWE and TE for liver fibrosis staging. Zhuang et al [27] showed that the diagnostic performance of SWE, namely the sensitivity and specificity, were higher in the diagnosis of F4 more than F2 and F3 which is almost similar to our results as noted in Table 4; SWE showed higher sensitivity, negative predictive value (NPV), and positive predictive value (PPV) in patients with F4 compared to the other patients when compared to tissue biopsy.

European Federation for societies for ultrasound in Medicine and Biology recommended the SWE to assess the degree of liver stiffness in patients with CLD secondary to hepatitis especially hepatitis $C$ [28].

The limitation of our study was the absent control group due to the difficulty to get a biopsy from a healthy person. Also, still, the US is operator dependent, and we didn't assess the inter-operator variability which of course further studies are needed to study this issue.

\section{Conclusion}

SWE shows almost similar diagnostic performance compared to fibroscan which considered recently as a main non-invasive tool in liver fibrosis staging with minimal tendency to overestimate the degree of fibrosis. SWE can be used with high performance as an alternative to fibroscan especially when fibroscan is not able to obtain adequate results as in obesity and massive free ascites. Also, SWE gives the operator a real-time visualization of the selected area with a large surface area compared to fibroscan.

\section{Abbreviations}

CLD: Chronic liver disease; NPV: Negative predictive value; PPV: Positive predictive value; SWE: Shear wave elastography; TE: Transient elastography; US: Ultrasound

\section{Authors' contributions}

$A M, A E$, and $M M$ share together in writing, editing, data collection, and statistical analysis of the research in almost the same way. All share together as operators almost equally in SWE and fibroscan. All authors read and approved the final manuscript.

\section{Funding}

The authors declare that this work has not received any funding.

Availability of data and materials

Available on request with the corresponding author.

\section{Ethics approval and consent to participate}

Ethics approval was taken from our departmental ethical committee (Radiology department-Ain shams university) with informed written consent that was taken from each patient.

\section{Consent for publication}

All patients included in this research gave written informed consent to publish the data contained within this study.

\section{Competing interests}

The authors declare that they have no competing interests.
Received: 3 December 2019 Accepted: 30 January 2020

Published online: 10 March 2020

\section{References}

1. O'Hara S, Hodson S, Hernaman C, Wambeek N, Olynyk J (2017) Concordance of transient elastography and shear wave elastography for measurement of liver stiffness. Sonography 4:141-145

2. Abd El Rihim AY, Omar RF, Fathalah W, El Attar I, Hafez HA, Ibrahim W (2013) Role of fibroscan and APRI in the detection of liver fibrosis: a systematic review and meta-analysis. Arab J Gastroenterol 14:44-50

3. Abdelmoneim S, Kamel S, Ahmed M, Naser A, Aly M (2013) Risk factors for progression of chronic hepatitis c infection to cirrhosis in rural Upper Egypt: a retrospective study. AAMJ 11:2

4. Ali Z, Zytoon A, Elsakhawy M, Algamal R (2018) Real-time shear wave elastography for assessing liver fibrosis in patients with chronic hepatitis C. Menoufia Medical Journal 31:538-543

5. Bravo AA, Sheth SG, Chopra S (2001) Liver biopsy. N Engl J Med 344:495500

6. Papastergiou V, Tsochatzis E, Burroughs A (2012) Non-invasive assessment of liver fibrosis. Ann Gastroenterol 25:218-231

7. Shiina T, Nightingale KR, Palmeri ML et al (2015) WFUMB guidelines and recommendations for clinical use of ultrasound elastography: part 1: basic principles and terminology. Ultrasound Med Biol 41:1126-1147

8. Sandrin L, Fourquet B, Hasquenoph J-M et al (2003) Transient elastography: a new noninvasive method for assessment of hepatic fibrosis. Ultrasound Med Biol 29:1705-1713

9. Guibal A, Renosi G, Rode A et al (2016) Shear wave elastography: an accurate technique to stage liver fibrosis in chronic liver diseases. Diagn Interv Imaging 97:91-99

10. Tada T, Kumada T, Toyoda $\mathrm{H}$ et al (2015) Utility of real-time shear wave elastography for assessing liver fibrosis in patients with chronic hepatitis C infection without cirrhosis: comparison of liver fibrosis indices. Hepatol Res. 45(10):122-129

11. Song P, Mellema DC, Sheedy SP et al (2016) Performance of 2-dimensional ultrasound shear wave elastography in liver fibrosis detection using magnetic resonance elastography as the reference standard: a pilot study. J Ultrasound Med. 35(2):401-412

12. Barr RG, Ferraioli G, Palmeri ML et al (2016) Elastography assessment of liver fibrosis: society of radiologists in ultrasound consensus conference statement. Ultrasound 32:94-107

13. Lédinghen V, Vergniol J (2008) Transient elastography (FibroScan). Gastroenterol Clin Biol 3:58-67

14. Bedossa P, Poynard T (1996) The METAVIR Cooperative Study Group. An algorithm for the grading of activity in chronic hepatitis C. Hepatology 24 : 289-293

15. Wong W, Vergniol J, Wong GL et al (2010) Diagnosis of fibrosis and cirrhosis using liver stiffness measurement in nonalcoholic fatty liver disease. Hepatology 51:454-462

16. Pavlov CS, Casazza G, Nikolova D et al (2015) Transient elastography for diagnosis of stages of hepatic fibrosis and cirrhosis in people with alcoholic liver disease. Cochrane Database Syst Rev. 1:CD010542

17. Ferraioli G, Wong V, Castera L et al (2018) Liver ultrasound elastography: an update to the World Federation for the US in medicine and biology guidelines and recommendations. Ultrasound Med Biol. 44(12):2419-2440

18. European Association for the Study of the Liver (2011) EASL clinical practice guidelines: management of hepatitis $C$ virus infection: European association for the study of the liver. J Hepatol 55:245-264

19. Castera L, Foucher J, Bernard P et al (2010) Pitfalls of liver stiffness measurement: a 5-year prospective study of 13,369 examinations. Hepatology 51:828-835

20. Foucher J, Chanteloup E, Vergniol J et al (2006) Diagnosis of cirrhosis by transient elastography (FibroScan): a prospective study. Gut 55:403-408

21. Zeng J, Zheng J, Huang Z et al (2017) Comparison of 2D shear wave elastography and transient elastography for assessment of liver fibrosis in chronic hepatitis B. Ultrasound Med Biol. 43(8):1563-1570

22. Ryu H, Ahn SJ, Yoon JH, Lee JM (2019) Reproducibility of liver stiffness measurements made with two different 2-dimensional shear wave elastography systems using the comb-push technique. Ultrasonography 38:246-254

23. Roccarina D, Prat LI, Guerrero M et al (2018) Comparison of ElastPQ shear wave elastography (Elast PQ-SWE) and fibroscan transient elastography (F-TE) for liver fibrosis staging in patients with NAFLD. J Hepatol 68(1):s554 
24. Leung V, Shen J, Wong V et al (2013) Quantitative elastography of liver fibrosis and spleen stiffness in chronic hepatitis B carriers: comparison of shear-wave elastography and transient elastography with liver biopsy correlation. Radiology 269:910-918

25. Ferraioli G, Tinelli C, Zicchetti M et al (2012) Reproducibility of real-time shear wave elastography in the evaluation of liver elasticity. Eur J Radiol 81: 3102-3106

26. Deffieux T, Gennisson JL, Bousquet $L$ et al (2015) Investigating liver stiffness and viscosity for fibrosis, steatosis and activity staging using shear wave elastography. J Hepatol 62(2):317-324

27. Zhuang Y, Ding H, Zhang Y, Sun H, Xu C, Wang W (2017) Two-dimensional shear-wave elastography performance in the noninvasive evaluation of liver fibrosis in patients with chronic hepatitis B: comparison with serum fibrosis indexes. Radiology 283(3):873-882

28. Bamber J, Cosgrove D, Dietrich CF et al (2013) EFSUMB guidelines and recommendations on the clinical use of ultrasound elastography. Part 1: basic principles and technology. Ultraschall Med 34:169-184

\section{Publisher's Note}

Springer Nature remains neutral with regard to jurisdictional claims in published maps and institutional affiliations.

\section{Submit your manuscript to a SpringerOpen ${ }^{\odot}$ journal and benefit from:}

- Convenient online submission

- Rigorous peer review

- Open access: articles freely available online

- High visibility within the field

- Retaining the copyright to your article

Submit your next manuscript at $\boldsymbol{\nabla}$ springeropen.com 